\title{
Self-Sustained Localized Structures in a Boundary-Layer Flow
}

\author{
Yohann Duguet, ${ }^{1}$ Philipp Schlatter, ${ }^{2}$ Dan S. Henningson, ${ }^{2}$ and Bruno Eckhardt ${ }^{3}$ \\ ${ }^{1}$ LIMSI-CNRS, UPR 3251, F-91403 Orsay, France \\ ${ }^{2}$ Linné FLOW Centre, KTH Mechanics, Osquars Backe 18, SE-100 44 Stockholm, Sweden \\ ${ }^{3}$ Fachbereich Physik, Philipps-Universität Marburg, Renthof 6, D-35032 Marburg, Germany and J. M. Burgerscentrum, \\ Delft University of Technology, 2628 CD Delft, The Netherlands
}

(Received 17 June 2011; published 25 January 2012)

\begin{abstract}
When a boundary layer starts to develop spatially over a flat plate, only disturbances of sufficiently large amplitude survive and trigger turbulence subcritically. Direct numerical simulation of the Blasius boundary-layer flow is carried out to track the dynamics in the region of phase space separating transitional from relaminarizing trajectories. In this intermediate regime, the corresponding disturbance is fully localized and spreads slowly in space. This structure is dominated by a robust pair of low-speed streaks, whose convective instabilities spawn hairpin vortices evolving downstream into transient disturbances. A quasicyclic mechanism for the generation of offspring is unfolded using dynamical rescaling with the local boundary-layer thickness.
\end{abstract}

DOI: 10.1103/PhysRevLett.108.044501

PACS numbers: 47.61.Jd, 47.10. $-\mathrm{g}, 68.08 . \mathrm{Bc}$

Subcritical bifurcations occur in physical systems as diverse as pattern-forming reacting systems [1], fluid flows [2], magnetic reconnection [3], and accretion disks [4]. They require finite-amplitude triggers to transition from one state to the other. Recent progress has been made via the possibility of identifying the edge state [5], the asymptotical fate of all perturbations at the critical threshold amplitude. In spatially extended systems, transition is connected to localized edge states. A common feature of all former investigations is the spatial homogeneity of the underlying base state. Spatially developing media form a much wider class of systems, a prime example being the Blasius boundary-layer flow, which captures most of the features of flows around smooth bodies. We identify here the corresponding localized structures in that boundarylayer flow and describe their spatial development as they advect downstream, the process by which they are selfsustained, and the dynamics inside the structure.

The Blasius boundary layer forms when a fluid of nonzero viscosity flows over a flat surface [6]. There is no natural length scale to the problem except the distance $\ell$ from the leading edge of the plate. Viscous diffusion of the vorticity generated at the wall makes the boundary layer grow in height, starting from the leading edge. A selfsimilar laminar solution of the two-dimensional incompressible boundary-layer equations can be found in terms of the rescaled variable $\eta=y\left(U_{\infty} / \nu \ell\right)^{1 / 2}$, where $y$ is the wall-normal distance to the plate, $U_{\infty}$ is the velocity far above the plate, and $\nu$ is the kinematic viscosity of the fluid [6]. A local Reynolds number can be defined as $\operatorname{Re}_{\delta^{*}}(\ell)=$ $U_{\infty} \delta^{*}(\ell) / \nu$, where $\delta^{*}=\int_{0}^{\infty}\left[1-u(y) / U_{\infty}\right] d y=O\left(\ell^{1 / 2}\right)$ is the displacement thickness, with $u(y)$ the streamwise velocity (see Fig. 1). The self-similar Blasius solution represents the laminar base flow, which is linearly stable for $\operatorname{Re}_{\delta^{*}} \leq \operatorname{Re}_{c} \approx 520$ [2]. There are two possible routes for the laminar-turbulent transition: in a weakly noisy environment, classical transition occurs supercritically for $\operatorname{Re}_{\delta^{*}} \geq \operatorname{Re}_{c}$, where the base flow is unstable to infinitesimal disturbances. In the presence of stronger noise, a subcritical transition can occur further upstream via a bypass scenario: the base flow is unstable to finiteamplitude disturbances only. Subcritical transition occurs also in pipe flow and plane Couette flow [7], where nonlinear coherent structures were identified on the phase space boundary $\Sigma$ separating laminar and turbulent motion. The technique for the identification of critical

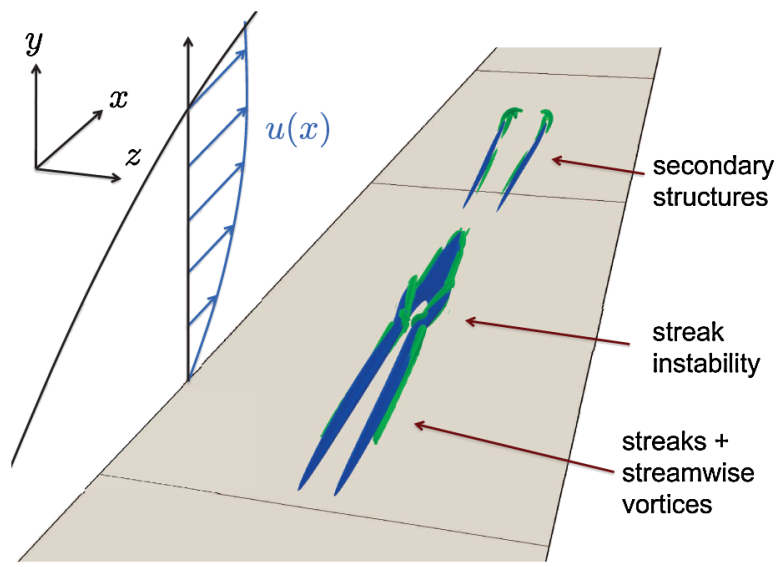

FIG. 1 (color online). Snapshot of the localized structure at $t=1050$. Isosurfaces of streamwise velocity perturbation $u=$ -0.08 [dark gray (blue)] and $\lambda_{2}=-10^{-5}$ [light gray (green)]. The robust core of the structure consists of streaks and streamwise vortices, indicated as well as the early stage of a streak instability. Out of the streak instability grow secondary structures that move downstream and dissipate. The spanwise lines correspond to $x=600,800$, and 1000 . The developing boundary layer is sketched out-of-scale on the left of the figure. 
disturbances proposed in Refs. [5,8,9] is based on identifying initial conditions which neither return to laminar nor become turbulent in the course of time, but instead approach the edge state, the relative attractor within this subspace. The associated velocity field in physical space is three-dimensional and combines streamwise streaks (spanwise modulations of the streamwise velocity) and streamwise vortices into a single stationary or traveling structure via a nonlinear self-sustaining process (SSP) [10,11]. This state, unstable by construction, is transiently approached by all trajectories starting in the vicinity of $\Sigma$. Edge states [12-15] become localized and unsteady when the domain is extended in all homogeneous directions. The situation for the Blasius boundary layer is a step up in complexity, both conceptually and technically: the spatial development implies that the control parameters vary along a trajectory, and the flow is not confined in the wall-normal direction. A recent calculation in a narrow periodic domain demonstrates the applicability of edge tracking in a spatially developing flow [16]. The introduction of a fixed length scale is, however, incompatible with the localization and the spatial spreading of the disturbance which follows from the spatial expansion of the flow. In order to capture the physically relevant dynamics, we use a sufficiently wide domain such that we can track fully localized critical disturbances in a realistic external boundary-layer flow.

Direct numerical simulation of the incompressible Navier-Stokes equations is performed by a spectral method using Fourier modes in the streamwise $x$ and spanwise $z$ directions and Chebyshev polynomials in the wall-normal direction $y$. The boundary conditions for the velocity field $(u, v$, and $w)$ are no-slip and impermeability on the plate $(y=0)$ and vanishing wall-normal derivatives at a finite yet large enough value of $y=L_{y}$. The use of Fourier modes implies periodicity in $x$ and $z$. However, because $\delta^{*}$ increases with $x$, making streamwise periodicity unsuitable, a fringe region is incorporated ahead of the downstream edge of the computational domain, in which artificial volume forcing dampens any disturbance [17]. The displacement thickness at $x=0$ is $\delta^{*}=\delta_{0}^{*}$, chosen such that $\operatorname{Re}_{\delta_{0}^{*}}=300$. We use a computational domain of size $\left(L_{x}, L_{y}, L_{z}\right)=(3000,60,100)$ in units of $\delta_{0}^{*}$, the fringe region starting at $x=x_{f}=2700$. This implies that $\delta^{*}$ grows by a factor of 5 over the length of the domain. Time $t$ is expressed in units of $\delta_{0}^{*} / U_{\infty}$. The numerical resolution is $\left(N_{x}, N_{y}, N_{z}\right)=(3072,201,256)$ spectral modes, and the phase space has dimension $N=$ $2 N_{x} N_{y} N_{z} \approx 3.16 \times 10^{8}$.

The dynamics on $\Sigma$ is tracked using the bisection method of Ref. [9]. A spatially localized initial perturbation to the laminar base flow is imposed near $x=0$, similar to the one used in Ref. [12]. It has the spanwise symmetry $(u, v, w)(x, y, z)=(u, v,-w)(x, y,-z)$. Its amplitude is then adjusted until the corresponding phase space trajectory neither reaches the base state nor the turbulent attractor. The practical criterion for deciding whether the regime is turbulent or laminar is based on adequate thresholding of two volume-integrated quantities: the streamwise vorticity $\omega_{x}=\left[\int\left(\partial_{y} w-\partial_{z} v\right)^{2} d v / \int d v\right]^{1 / 2}$ and the wallnormal vorticity $\omega_{y}=\left[\int\left(\partial_{z} u-\partial_{x} w\right)^{2} d v / \int d v\right]^{1 / 2}$ (where $d v=d x d y d z$ ). $\omega_{x}$ is a direct measure of the amplitude of streamwise vortices, while the temporal fluctuations of $\omega_{y} \approx\left|\partial_{z} u\right|$ reflect the amplitude of the streaks, both of those quantities vanishing for the laminar flow. In a spatially parallel flow, such a bisection can in principle be carried out ad eternum when periodic boundary conditions are used, while for the Blasius case the effective length $x_{f}$ is the limiting parameter. Edge tracking also proved robust for values of $\operatorname{Re}_{\delta^{*}}$, where the base flow is no longer linearly stable.

For $t \geq 500$, the flow has lost the memory of the initial perturbation, suggesting that a relative attractor is being approached. The spatial structure stays fully localized but grows slowly in all directions. Within it, we identify a robust core (the upstream part of the structure) that persists with relatively little time dependence and secondary structures downstream with larger fluctuations in time. The robust core is best isolated at $t \approx 1050$ (Fig. 1). It features at all times a pair of elongated low-speed streaks. These streaks support convective instabilities in the form of timedependent wavy modulations [18]. Visualization of the vortices using the $\lambda_{2}$ vortex identification criterion [19] reveals longitudinal vortices staggered on the flanks of the streaks, as well as hairpin-shaped vortices forming precisely above the region where streaks pinch. The temporal robustness of this pinching structure suggests that it is a quasiequilibrium state of the flow. The process is comparable to that described in Ref. [20]; however, it does not require continuous forcing, only adequate initial triggering. Note also the different symmetries compared to Waleffe's SSP [10], where sinusoidal streak instabilities are found that do not give rise to hairpin vortices. However, the global structure is highly unsteady in time as the secondary structures undergo large temporal fluctuations, which will be characterized in detail below.

The slow growth of the boundary-layer thickness $\delta^{*}(x)$ suggests the use of rescaled variables for a description of the temporal dynamics. This is accomplished by rescaling all space and time variables by a factor of $\delta_{0}^{*} / \delta(t)$, where $\delta(t)$ refers to the local displacement thickness $\delta^{*}$ at the "location" $x(t)$ of the structure at time $t$. Interpreted as the maximum shear at the wall, $x(t)$ is found in practice by locating the maximum over $x$ of $\delta \omega_{z}(y=0)$, where we have defined

$$
\delta \omega_{z}(x, y, t)=\omega_{z}(x, y, z=0, t)-\left\langle\omega_{z}\right\rangle_{z}(x, y, t) .
$$

Velocities are unaffected by the rescaling; thus, the convection speed $\dot{x}(t)$ at the wall is a constant independent of the rescaling. Taking into account the $\delta=O\left(\ell^{1 / 2}\right)$ development of the Blasius solution, we anticipate that 

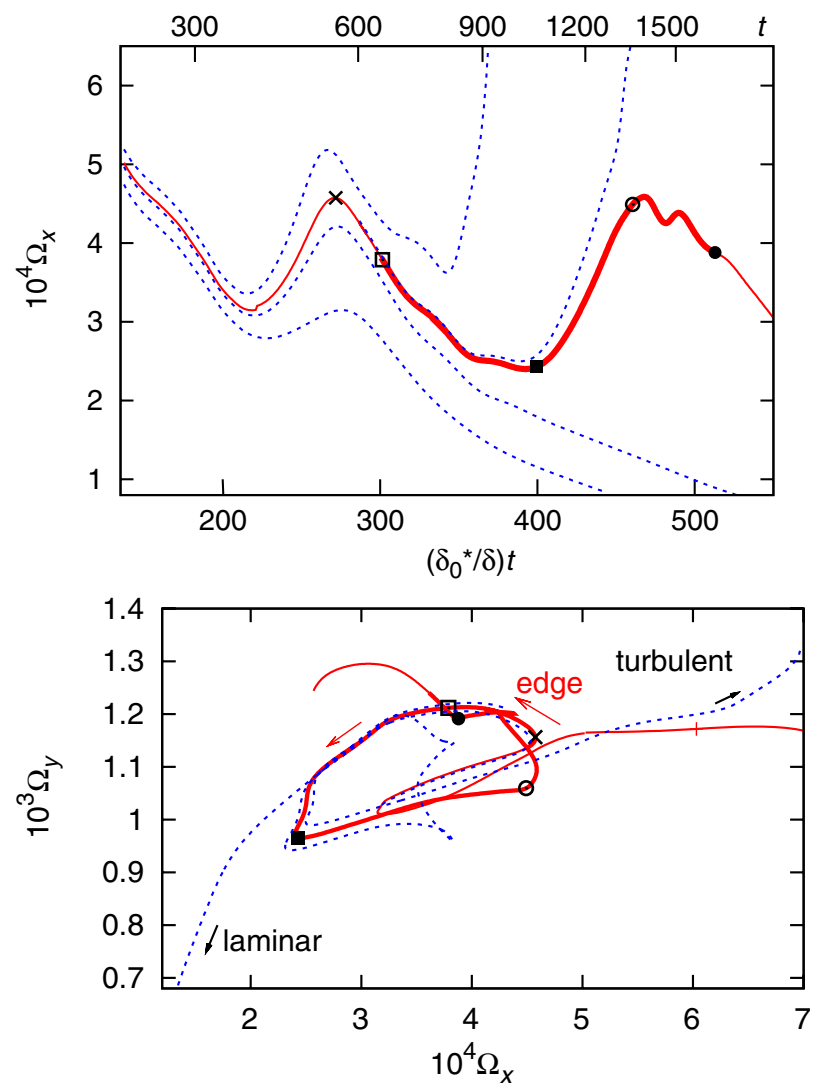

FIG. 2 (color online). Top: Rescaled vorticity $\Omega_{x}$ vs $t$ and $\left(\delta_{0} t / \delta^{*}\right)$. Bottom: Projection on the $\left(\Omega_{x}, \Omega_{y}\right)$ space. The edge trajectory is plotted as solid red lines, and the diverging trajectories are plotted in dotted blue lines. The symbols corresponds to times $t=550$ (cross), 651 (open square), 1050 (filled square), 1350 (open circle), and 1640 (filled circle). The cyclical phase $650 \leq t \leq 1640$ is plotted in thicker red lines.

$\delta(t) / \delta_{0}^{*} \approx\left(1+t / t_{0}\right)^{1 / 2}$ and find a good fit with $t_{0} \approx$ 177.8. Vorticities are, however, rescaled by $\left[\delta(t) / \delta_{0}^{*}\right]$. The dimensions of the numerical domain are now timedependent in the rescaled variables; hence, a volumetric correction factor $\left[\delta(t) / \delta_{0}^{*}\right]^{3}$ must be incorporated into the new integral vorticities $\Omega_{i}=\left(\delta_{0}^{*} / \delta\right)^{1 / 2} \omega_{i}(i=x, y)$. We show $\Omega_{x}$ plotted vs time in Fig. 2 (top), and a twodimensional phase space projection $\left(\Omega_{x}, \Omega_{y}\right)$ is shown in Fig. 2 (bottom). In the rescaled variables, it becomes clear that, past the transient phase, the dynamics is cyclical in time on a slow time scale of $\approx 200 \delta / U_{\infty}$. The four snapshots displayed in Fig. 3 cover one period of the quasicycle, where the first and the last snapshot are taken at approximately the same position in the cycle. Note that the upstream robust core shows strong similarities at those 2 times, although the downstream secondary structures exhibit differences. In the four snapshots, we see the tail of the robust core slowly lengthening during the cycle, as well as the detachment and disappearance of secondary structures downstream. All snapshots shown are rescaled in units of $\delta(t)$, and the temporal evolution of the robust core during the period depicted implies that we have not reached an asymptotic self-similar edge state. This also means that we cannot expect the next period of the cycle to strictly retrace the path traced by the thick red curve in Fig. 2. Scrutinizing the dynamics of low-speed streaks and hairpin vortices in Fig. 3 and from a corresponding animation [21] also suggests a cyclic succession of events. At $t \approx$ 650 , that region downstream is dominated by a pair of lowspeed streaks. As time evolves, those streaks vanish slowly. This corresponds to the phase where both $\Omega_{x}$ and $\Omega_{y}$ decay. Note that the whole disturbance would have dissipated if the amplitude at $t=0$ had been slightly lower (see the relaminarizing trajectory in Fig. 2). Both $\Omega_{x}$ and $\Omega_{y}$ increase again from $t=1050 \mathrm{on}$, meaning that vorticity is produced, corresponding to the formation of hairpin vortices apparent in Figs. 1 and 3. This increase of vorticity could go on all the way towards the turbulent spot regime (see the transitioning trajectory in Fig. 2); yet, in the critical regime, $\Omega_{x}$ decreases again from $t \approx 1350$ on. New low-speed streaks form downstream, and the process can start again. An early version of the same cycle is also visible in Fig. 2 (top) and in the animation for $300 \leqq t \lesssim$ 600. The quasicyclic variations in Fig. 2 are thus associated to the streak dissipation or regeneration events downstream of the robust core. The root of the instability responsible for the regeneration of these secondary structures is not local but has to be sought upstream, precisely where hairpin vortices are generated. Isosurfaces of negative $\lambda_{2}$ show that neighboring streamwise vortices located near the pinching region can reconnect to form hairpin-shaped vortices lifted up above the low-speed streaks [22,23]. Hairpin vortices form as the finite-amplitude outcome of streak instabilities of the subharmonic-sinusoidal or varicose types [24]. In order to understand how vorticity can be transferred from this region upstream to the region downstream while the structure moves as a whole, we quantify the velocities of the vortical perturbations. Space-time

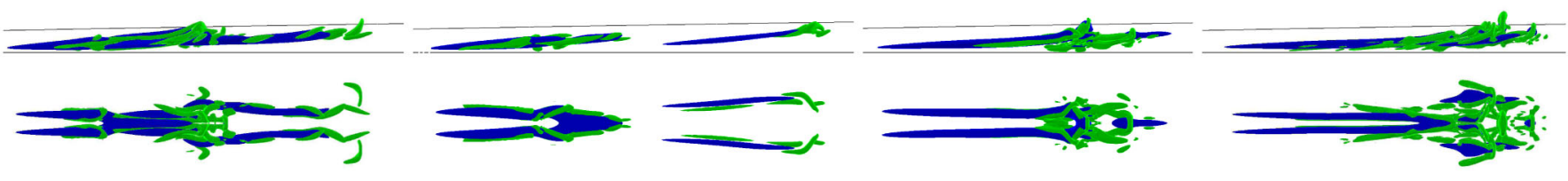

FIG. 3 (color online). Side and top view of the quasicycle in rescaled spatial units. Four snapshots are shown, corresponding to the nonrescaled times $t=650,1050,1350$, and 1640 marked in Fig. 2 (from left to right), with the same color code as in Fig. 1. The $x$ direction is squeezed by a factor of 2 for better visibility, with the flow from left to right. 


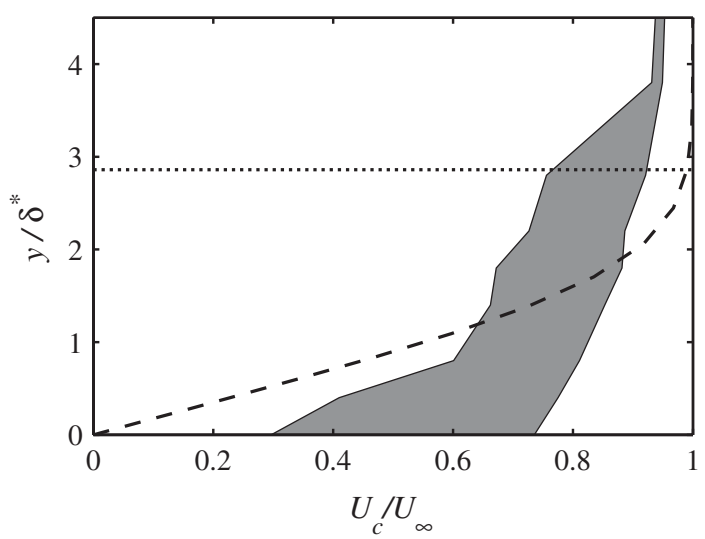

FIG. 4. Convection velocity $U_{c} / U_{\infty}$ of perturbations vs $y / \delta^{*}$ (shaded area). The Blasius profile is shown (dashed line). The dotted line represents the position at which the Blasius profile represents $99 \%$ of $U_{\infty}$.

diagrams for $\delta \omega_{z}(x, t)$ were computed for all values of the reduced wall-normal coordinate $y^{*}=y / \delta^{*}(x)$. Velocities can be extracted from these diagrams via linear interpolation, yielding the quantity $U_{c}\left(y^{*}\right)$ shown in Fig. 4 . The lowest velocity is the signature of the streaks near the wall, the others corresponding to convective streak instabilities [18]. Both depart clearly from the Blasius profile, the departure being maximal closest to the wall, as in the turbulent regime [25]. Importantly, the hairpin vortices, located above the streaks, travel fast enough to overtake the core region. When far enough above the wall (at $t=$ 650 and 1640), the vortices furthest on the side have positive spanwise vorticity and can induce a local deficit of streamwise velocity below their heads. New low-speed streaks are thus born, replacing the ones that have decayed earlier, while vortices move ahead of the structure and dissipate. Figure 3 shows that the ejection and transport of vortices give rise to a fluctuating leading edge position, whereas the robust core moves steadily.

Using the technique of edge tracking, we have identified a relatively simple localized structure able to sustain itself against viscous decay in a spatially developing boundary layer. As it travels downstream, this structure stretches, adapting itself to the local characteristic length scale $\delta^{*}$ of its environment. It is plausible that asymptotically long edge tracking would lead to a perfectly self-similar edge state. The root for the sustenance of the structure investigated here is located upstream, at the junction of two lowspeed streaks. For localized edge states in pipe flow $[13,15]$, strong spatial coherence suggestive for an SSP [11] was found solely near the trailing edge, comparable in energy to known (short-wavelength) traveling wave solutions. The fluctuating leading edge appeared only as a diffusive front, and the dynamic connection between the two fronts remained obscure. In the present case of the boundary layer, we also distinguish a robust and strongly coherent structure upstream from a diffusive one down- stream. The dynamic connection between the two fronts is now clear: the fluctuating dynamics of the downstream front can simply be interpreted as the transient outcomes from convective instabilities occurring upstream and advected faster. These observations will prove useful for a better understanding of the transition to turbulence via the formation of localized patterns.

Computer time provided by the Swedish National Infrastructure for Computing (SNIC) is gratefully acknowledged. D. H. thanks the A. von Humboldt foundation, and B. E. and D. H. thank the GFD program at WHOI for support.

[1] E. Knobloch, Nonlinearity 21, T45 (2008).

[2] P. J. Schmid and D.S. Henningson, Stability and Transition in Shear Flows (Springer, New York, 2001).

[3] P. A. Cassak, J. F. Drake, M. A. Shay, and B. Eckhardt, Phys. Rev. Lett. 98, 215001 (2007).

[4] F. Rincon, G. I. Ogilvie, and M. R. E. Proctor, Phys. Rev. Lett. 98, 254402 (2007).

[5] J. D. Skufca, J. A. Yorke, and B. Eckhardt, Phys. Rev. Lett. 96, 174101 (2006).

[6] H. Blasius, Z. Math. Phys. 56, 1 (1908).

[7] B. Eckhardt, T. Schneider, B. Hof, and J. Westerweel, Annu. Rev. Fluid Mech. 39, 447 (2007).

[8] S. Toh and T. Itano, J. Fluid Mech. 481, 67 (2003).

[9] T. Schneider, B. Eckhardt, and J. Yorke, Phys. Rev. Lett. 99, 034502 (2007).

[10] F. Waleffe, Phys. Fluids 9, 883 (1997).

[11] F. Waleffe, J. Fluid Mech. 435, 93 (2001).

[12] Y. Duguet, P. Schlatter, and D.S. Henningson, Phys. Fluids 21, 111701 (2009).

[13] F. Mellibovsky, A. Meseguer, T. M. Schneider, and B. Eckhardt, Phys. Rev. Lett. 103, 054502 (2009).

[14] T. Schneider, D. Marinc, and B. Eckhardt, J. Fluid Mech. 646, 441 (2010).

[15] Y. Duguet, A. P. Willis, and R. R. Kerswell, J. Fluid Mech. 663, 180 (2010).

[16] S. Cherubini, P. D. Palma, J.-C. Robinet, and A. Bottaro, Phys. Fluids 23, 051705 (2011).

[17] M. Chevalier, P. Schlatter, A. Lundbladh, and D. S. Henningson, KTH Mechanics Report No. TRITA-MEK 2007:07, 2007.

[18] L. Brandt, C. Cossu, J.-M. Chomaz, P. Huerre, and D. S. Henningson, J. Fluid Mech. 485, 221 (2003).

[19] J. Jeong and F. Hussain, J. Fluid Mech. 285, 69 (1995).

[20] T. Duriez, J. L. Aider, and J. E. Wesfreid, Phys. Rev. Lett. 103, 144502 (2009).

[21] See Supplemental Material at http://link.aps.org/ supplemental/10.1103/PhysRevLett.108.044501 for animation of the nonrescaled flow field, with the same color code as in Fig. 1.

[22] M. S. Acarlar and C. R. Smith, J. Fluid Mech. 175, 43 (1987).

[23] R. J. Adrian, Phys. Fluids 19, 041301 (2007).

[24] P. Andersson, L. Brandt, A. Bottaro, and D.S. Henningson, J. Fluid Mech. 428, 29 (2001).

[25] J. Kim and F. Hussain, Phys. Fluids A 5, 695 (1993). 